\title{
LAS BIBLIOTECAS CENTRALES DE COMUNIDADES AUTÓNOMAS DEL ESTADO ESPAÑOL. SU COLECCIÓN DE MATERIALES
}

\author{
Juan José Fuentes Romero*
}

Resumen: A partir de un intento de clarificación del concepto y realidad de las aqui llamadas Bibliotecas Centrales de Comunidades Autónomas, en el Estado español, se presentan las líneas generales en cuanto a la planificación del desarrollo de la colección, sus elementos integrantes, los materiales, la cuestión del Depósito Legal, la conservación y el expurgo.

Palabras clave: bibliotecas nacionales, colección de materiales, planificación de bibliotecas.

Abstract: Study on the denomination and chacteristics of the Central libraries of the Spanish Autonomous Communities, with a special focus on the collection planning.

Key words: national libraries, materials collection, library planning.

\section{Introducción}

Parece conveniente, para empezar, el dejar claro qué queremos decir cuando hablamos de Biblioteca Central de Comunidad Autónoma.

Podríamos señalar, de entrada, que nos referimos a aquellas bibliotecas que, en cada Comunidad Autónoma del Estado español, desarrollan unas misiones que, en mayor o menor medida, son -0 debieran ser- equiparables, en su entorno, a las de una Biblioteca Nacional.

La cuestión, y ello se comprenderá fácilmente, se presenta compleja.

$\mathrm{Si}$, por una parte, hay ciertas semejanzas entre esta nueva tipología bibliotecaria y las tradicionalmente llamadas Bibliotecas Nacionales, evidente resulta que también existen enormes diferencias; no obstante, estos nuevos centros que han aparecido en el panorama bibliotecario y cultural español deben, con toda justicia, englobarse dentro del dominio de las Bibliotecas Nacionales.

A los efectos de este artículo, y sin entrar en mayores profundidades, utilizaremos la denominación de Biblioteca Central de Comunidad Autónoma (en adelante, BCC).

\section{Biblioteca Nacional y Centro del Sistema Nacional Bibliotecario}

Complejo resulta definir qué sea una Biblioteca Nacional, de modo que Line (1) afirma: "Resulta fácil pensar en la Biblioteca Nacional como una categoría bien establecida de bibliotecas que ocupan una posición similar y desarrollan simi-

* Universidad de A Coruña

Recibido: 26-1-2000. Segunda versión: 29-6-2000. 
lares papeles en la mayoría, si no en todos, los países. No obstante las Bibliotecas Nacionales son extraordinariamente difíciles de definir y presentan una gran variedad en su tamaño, naturaleza y funciones, y no sólo eso, sino que no existe una clara línea divisoria entre la planificación de una Biblioteca Nacional y la planificación nacional de bibliotecas, de modo que en los diversos países diversas bibliotecas pueden estar desempeñando en mayor o menor medida el papel de la Biblioteca Nacional»

Goodrum (2) al tratar el tema de las Bibliotecas Nacionales, propone dividir la enorme cantidad de ellas existente en tres grandes categorías.

La primera generación, de la que señala unos veinte ejemplos, comprende las $\mathrm{Bi}$ bliotecas Nacionales tradicionales y clásicas en las que automáticamente pensamos cuando usamos este término en el dominio de los estudios de biblioteconomía. Aparecieron antes del siglo XVIII y sus ejemplos conspicuos podrían ser las Bibliotecas Nacionales de España, Francia, Reino Unido, etc.

Su fundación deriva de las grandes bibliotecas reales o también de grandes colecciones privadas. Mediante el privilegio del Depósito Legal han desarrollado amplísimas colecciones de la literatura nacional, produciendo a partir de ellas las bibliografías nacionales y los catálogos colectivos; asimismo, son estas bibliotecas las encargadas de establecer las normas bibliográficas nacionales. Suelen ser los más importantes centros para la investigación bibliográfica del país.

La segunda generación de Bibliotecas Nacionales se extiende desde el período post-napoleónico a la segunda guerra mundial y engloba unos cincuenta ejemplos. Aunque parten de los modelos de la primera generación, tanto los desastres históricos como la competencia expansiva de las bibliotecas universitarias y públicas hizo que este grupo se autolimitara en su actuación y desarrollara sólo algunas funciones nacionales, principalmente en lo referido a la acumulación, en forma impresa, de la historia y de las realizaciones de la nación.

Goodrum, asimismo, subdivide este grupo en otros tres, el grupo latinoamericano, con espléndidos edificios pero inadecuados recursos; el grupo de las bibliotecas de $\mathrm{Ca}$ nadá, Australia y Nueva Zelanda que comienzan bien unidas a los gobiernos o como apoyo a sus parlamentos y, en tercer lugar, el grupo tipificado por Suiza, Israel y Alemania que se ha adecuado perfectamente a la competencia con otras importantes bibliotecas del país.

La tercera generación de estas Bibliotecas Nacionales está compuesta por las bibliotecas fundadas desde el final de la segunda guerra mundial. Son radicalmente diferentes en sus objetivos, dado que este tipo de bibliotecas son esencialmente cabeceras de redes provinciales o locales, con la misión de integrar un sistema. Funcionan normalmente como centros de formación bibliotecaria, prestan libros y mantienen la bibliografía nacional.

No obstante todo lo expuesto, Goodrum mantiene que existen diferencias entre una Biblioteca Nacional y el Centro Nacional del Sistema Bibliotecario.

Para Wilson (3) una Biblioteca Nacional es la que está legalmente constituida como tal y mantiene:

- El archivo impreso nacional con la ayuda del Depósito Legal

- La bibliografía nacional

- Una importante colección de literatura extrajera 
- El intercambio oficial y Depósito Legal de publicaciones

- La participación en las normas bibliográficas internacionales.

Frente a estas funciones de una Biblioteca Nacional, al Centro del Sistema Bibliotecario y de Información, siguiendo también a Wilson, le corresponderían las funciones siguientes:

1. Legislación de los servicios y de las normas para los diversos sectores bibliotecarios y de información.

2. Planificación y colocación de recursos respecto a todas las bibliotecas financiadas con fondos públicos: universitarias, gubernamentales y de investigación, públicas y escolares; asimismo, podría corresponderle a este centro la organización y dirección como sistema de uno o más de estos tipos de bibliotecas.

3. Desarrollo de redes cooperativas de recursos, incluyendo el préstamo, que necesariamente tienen un carácter formal cuando están basadas en las nuevas tecnologías de la información, en las telecomunicaciones o en cualquier otro sistema que implique gran cantidad de recursos.

4. Servicios de catalogación y otros servicios bibliográficos.

5. Mantenimiento de un saludable nivel profesional, tanto en lo referente al estatus de las asociaciones profesionales, como respecto a la cualificación, al reciclaje técnico y a las condiciones del servicio.

6. Promoción de un saludable comercio del libro y de la industria de la información que implique a las editoriales, a las librerías y a las empresas proveedoras de bases de datos para las redes electrónicas.

7. Establecimiento de enlaces con servicios comerciales bibliográficos y de recuperación de la información, a menudo ubicados fuera del pafs.

8. Relación con la normativa de telecomunicación y automatización del país.

9. Provisión de fondos para la investigación y el desarrollo.

10. Integración de la planificación del Sistema Bibliotecario y de Información con los objetivos - sociales, educacionales, culturales y económicos- de la comunidad y con los correspondientes a la comunidad informativa en su sentido más amplio (los medios de comunicación de masas).

Habría que decir, respecto a esta exhaustiva relación de las competencias que corresponderían al Centro Nacional del Sistema Bibliotecario y de Información, que tiene ciertos visos de ser más un desideratum que algo factible, pues no parece que, en ningún país de nuestro entorno cultural, exista realmente un centro que, tal como se requiere en el punto 2, se encargue de la planificación de absolutamente todos los subsistemas bibliotecarios (lectura pública, bibliotecas universitarias, etc.); por otra parte, algunos de los puntos señalados más serían competencias propias de la Biblioteca Nacional que de este centro, tales como el punto 4 (catalogación y servicios bibliográficos), el 7 (enlaces con servicios comerciales y bibliográficos, incluso de fuera del país) y el 8 (normativa de telecomunicación y automatización del país).

Es importante reseñar estas cuestiones que, aunque en Wilson están planteadas de manera puramente teórica, conducen, cuando eso se da en la realidad, a una cierta con- 
fusión entre los roles que deben desarrollar cada tipo de centro a los que nos estamos refiriendo.

\section{Las Bibliotecas Centrales de Comunidades Autónomas}

Si partimos de los tres tipos de Bibliotecas Nacionales de los que habla Goodrum, veremos que, en cuanto a las características que se dan en las BCC, aparecen algunas notas específicas propias de una Biblioteca Nacional, aunque también es cierto que faltan otras.

En primer lugar, y ello es más que evidente, las BCC del Estado español no representan a un Estado; es decir, pueden tener la conceptualización de «Nacional», pero su ámbito de competencias no va más allá de la Comunidad Autónoma correspondiente; la cuestión que conlleva el uso o no uso del calificativo «nacional» en este caso, como se comprenderá fácilmente, es de índole política, en modo alguno bibliotecaria, y surge del entendimiento de que Nación y Estado sean, o no, términos equiparables. Tema éste, obvio es, que en modo alguno corresponde a los límites y alcances del presente artículo.

Ahora bien, y este es el segundo punto que tomamos en consideración, no es menos cierto que, dentro de sus respectivas Comunidades, cumplen algunas funciones que, de suyo, son características propias de lo que tradicionalmente se ha venido entendiendo como «Biblioteca Nacional».

Sin excepción, en todas las BCC se señalan una serie de competencias que vamos a comentar y, para las cuales, pueden servirnos de modelo las recogidas en la Ley 8/1986 (4) de Bibliotecas de Aragón que, en su artículo 5, cita las siguientes:

«Recoger, conservar y difundir toda creación impresa, sonora, visual de Aragón y de los autores aragoneses producida en la Comunidad Autónoma o que hagan referencia a ella. A tal fin, recibirá, al menos, un ejemplar de las obras sujetas a depósito legal, en la forma que reglamentariamente se determine.

Ser depositaria de los fondos bibliográficos que sean donados o entregados en depósito a la Administración.

Elaborar y difundir la información bibliográfica sobre la producción editorial aragonesa.

Elaborar, en colaboración con el resto de las bibliotecas del sistema, el Catálogo Colectivo y las formas de consulta del mismo.

Establecer relaciones de colaboración e intercambio con otros sistemas bibliotecarios nacionales o extranjeros (5)».

Si analizamos, siquiera sea brevemente, estos puntos, veremos que se corresponden totalmente con las características propias y esenciales de una Biblioteca Nacional de corte tradicional; es decir, podemos afirmar con pleno convencimiento y absoluta propiedad en lo que estamos diciendo, que nos encontramos ante una nueva tipología de bibliotecas que, con independencia de que requieran o no para sí el calificativo de «nacional», tanto por su estructuración como por las funciones que ha de realizar son verdaderamente Bibliotecas Nacionales en el ámbito de sus respectivas Comunidades.

Paralelamente al esquema que propone Wilson de separación entre las Bibliotecas Nacionales y los Centros Nacionales del Sistema Bibliotecario, (diferenciación ésta 
que, en la práctica, resulta ser la más operativa) en las diversas Comunidades Autónomas se ha tendido a un modelo semejante, de modo que existe una dualidad entre la «Biblioteca Nacional» y el «Centro Nacional del Sistema Bibliotecario y de Información», con independencia del nombre concreto que reciba este centro en el organigrama bibliotecario de cada Comunidad Autónoma, de modo que, siguiendo con el ejemplo de Aragón (que, sin excesivas variantes, se repite en las demás Comunidades Autónomas) en el Decreto 202/1998 de dicha Comunidad se establece que, dentro del Departamento de Educación y Cultura, se crea el Servicio del Libro y Bibliotecas, al que corresponde la gestion de las competencias del Departamento en materia de investigación, depósito, creación, mantenimiento, promoción, publicación y difusión del patrimonio documental y bibliográfico contenido en cualquier tipo de soporte gráfico y audiovisual; la política del libro y la creación literaria; la atención de las Bibliotecas Provinciales... y de las Bibliotecas Municipales integradas en la Red Bibliotecaria de la Comunidad Autónoma de Aragón, así como de la Biblioteca de Aragón con los fondos netamente aragoneses integrados en el Instituto Bibliográfico Aragonés.

Nos encontramos, pues, ante una estructura bimembre en la cual el Centro $\mathrm{Na}$ cional del Sistema Bibliotecario y de Información (sea cual sea, repetimos, el nombre que este centro reciba en cada Comunidad Autónoma) ejerce las competencias puramente administrativas y de gestión referidas a todo el Sistema Bibliotecario, mientras a la Biblioteca Central (sea cual sea, también, el nombre que reciba) le corresponden las competencias propias y específicas de una Biblioteca Nacional; en el caso de Aragón, y aquí sí se producen diferencias nominales o conceptuales con otras Autonomías españolas, de la Biblioteca Central depende el Instituto Bibliográfico Aragonés.

\section{Características y fines de su colección de materiales}

Hasta aquí hemos tratado de dilucidar (y más bien dirfamos «plantear» la cuestión) referente a la denominación de estos centros; hemos señalado los caracteres esenciales que competen a una Biblioteca Nacional, viendo que, sin forzar excesivamente la cuestión, los más importantes de ellos se dan en las Bibliotecas Centrales de Autonomías y, finalmente, hemos tratado el tema de la dualidad que plantea la existencia de un Centro Administrativo y de Gestión y, dependiente de éste, la Biblioteca Nacional en sí misma.

Ahora vamos a ceñirnos a la Biblioteca Central de Comunidad Autónoma en sí misma, a tratar de ver su papel en una sociedad en que los cambios de toda índole (políticos, sociales, económicos, tecnológicos...) no son rápidos, sino vertiginosos.

Como punto de partida, podríamos asumir y aplicar a las BCC el principio básico que Morholt (6) establece para cualquier biblioteca actual, con independencia de su tipología: «Las tecnologías de redes y de recursos distribuidos de información, los programas adecuados para establecer vínculos entre recursos de información y el movimiento hacia sistemas cinemáticos e interactivos van a cambiar lo que son las bibliotecas y lo que los bibliotecarios hacen. Necesitamos planificar el cambio, de modo que se ponga el énfasis en que, de ser conservadores de libros, debemos llegar a ser quienes guíen a través del universo del conocimiento"

Partiendo, obviamente, de Ranganathan, Gorman (7) ha enunciado sus cinco nue- 
vas leyes de la Biblioteconomía, que de hecho no consisten sino en una puesta al día de las leyes del bibliotecario hindú; estas leyes son:

1. Las bibliotecas sirven a la humanidad.

2. Respetan todas las formas mediante las cuales se comunica el pensamiento.

3. Usan las tecnologías inteligentemente para mejorar sus servicios.

4. Protegen el libre acceso al conocimiento.

5. Honran el pasado y crean el futuro.

Evidentemente, se trata de una relación de principios aplicables a cualquier biblioteca de hoy día, pero tal vez dichos principios son excepcionalmente aplicables a las Bibliotecas Centrales de Comunidad; pensemos, sin entrar en mayores profundidades, en todo lo que implica el punto 5: «Honran el pasado y crean el futuro", y decimos esto porque, si hay un tipo de bibliotecas que de suyo pueda desarrollarlo, éste es, sin duda, el que engloba a las Bibliotecas Nacionales, sea cual sea el grupo en que queramos incardinarlas o la tipología a la que estimemos que pertenecen.

Concurren en las BCC, por una parte, la obligación de conservar los soportes que conlleven información (en el más extenso sentido de la palabra) respecto a su Comunidad, pero esa conservación sólo es lógicamente válida si sirve para la difusión.

Esto, que es algo tópico y esencial en el ser de cualquier biblioteca, se ve en estos días complicado por la avalancha de cambios que se está produciendo; en este sentido, Mc Gowan (8) señala una serie de tendencias, tales como:

1. La naturaleza cambiante del usuario de la biblioteca, como resultado de las nuevas posibilidades de acceso remoto y de la mejora en la entrega de documentos.

2. La tecnología de las comunicaciones, que favorece la posibilidad de compartir recursos - la «biblioteca global en la aldea global»-, aunque en este caso, Mac Gowan cita a Line (9) quien llama la atención sobre cómo esta tecnología, a su vez, puede llevar a un proceso creciente de competición.

3. El inicio del proceso de fusión de la recuperación de información bibliográfica relativa a un documento con el acceso al documento electrónico en sí mismo, señalándose diversos experimentos que permiten que la información esté electrónicamente disponible.

4. La continua discusión acerca de la conversión retrospectiva de catálogos.

5. La necesidad de revisar la legislación que cubre el Depósito Legal de los nuevos medios, así como la referente al derecho de copia.

Se trata ahora de un cambio tan absoluto que la cuestión esencial, afirma Betten (10), no es ya «qué ofrecemos», sino "cómo lo ofrecemos», afirmación ésta, qué duda cabe, de hondas resonancias macluhanianas, de modo que tal vez tengamos que admitir definitivamente que «el medio es el mensaje» (11).

Como cualquier otro tipo de bibliotecas, las BCC deben adaptarse a unos nuevos comportamientos en el almacenamiento, uso y difusión de la información, así como al tratamiento específico de los nuevos formatos; en este sentido, resulta esclarecedor el planteamiento que de esta cuestión realiza Brown (12); según él, en la sociedad se están produciendo actualmente las siguientes tendencias: 
1. Creciente uso de recursos electrónicos y disminución en el uso de materiales impresos.

2. Creciente interconectividad de los recursos.

3. Creciente número de usuarios de información que son remotos respecto a las instalaciones de la biblioteca.

4. Crecientes expectativas, por parte de los usuarios remotos, respecto al acceso a los catálogos en línea, a documentos en texto completo y a formatos multimedia.

Resulta evidente, y no añadimos nada nuevo, que las BCC han de replantearse su actuación en general si quieren sobrevivir, pues el "mercado» al que dirigen sus esfuerzos mira más hacia un futuro que ya es presente que hacia un pasado que ha concentrado, hasta ahora, la mayor parte de sus esfuerzos en la conservacion, en el almacenamiento, en la recogida de los materiales todos que, desde la información, se refieren a su área geográfica de trabajo.

En modo alguno estamos defendiendo que el almacenamiento y conservación de materiales deje de ser uno de los objetivos básicos de este tipo de centros, pues de manera esencial se constituyen en memoria física y real, que no meramente virtual, de cuanto, referido a su dominio de trabajo, acontece en el mundo de la información, del conocimiento, de la creatividad.

La evidencia, pues no otro es el caso, que estamos propugnando se refiere a los nuevos soportes, a las nuevas vías de acceso a la información, a las nuevas exigencias y necesidades de los usuarios, a un planteamiento nuevo respecto, esencial aunque no exclusivamente, a los criterios de selección, adquisición y mantenimiento de la colección de materiales.

Siendo como es la colección de materiales un elemento sine qua non en la mera existencia de cualquier tipo de bibliotecas, en el caso de las Bibliotecas Nacionales este componente llega a niveles de importancia muy superiores respecto a las demás. Se comprenderá que esto que decimos no es ninguna exageración, si tenemos en cuenta la esencial labor de conservación que, específicamente, compete a las BCC, lo que las hace tener unas características tan absolutamente peculiares.

\section{Planificación estratégica de la colección de materiales}

\subsection{Bases teóricas y objetivos de la planificación estratégica de la colección}

Para Koontz y Weihrich (13), «la planificación incluye seleccionar misiones y objetivos y las acciones para alcanzarlos; requiere tomar decisiones: es decir, seleccionar entre diversos cursos de acción futuros. Así, la planificación provee un enfoque racional para lograr los objetivos preseleccionados».

Sabido es que la planificación ha de ser general, es decir, aplicada a todos y cada uno de los elementos que configuran la vida y actuación de una organización, pero también, y de una forma simultánea, particular, lo que implica el estudio concreto de cada una de las secciones que conforman el organismo de que se trate.

En nuestro caso, y al tratar de las BCC, hemos de estudiar su planificación como 
bibliotecas en su conjunto, pero también la que se refiere a sus componentes, es decir, aplicada a la cuestión que ahora tratamos, la planificación de la colección de materiales.

La política de desarrollo de la colección (14) implica el disponer de una amplia visión de la colección considerada en sí misma y responde a cuestiones tales cómo ¿cuál es la misión fundamental de la biblioteca? ¿qué temas hay que reunir y hasta qué nivel de profundidad? ¿qué tipos de soportes hay que incluir en la colección y cuál ha de ser el balance entre estos soportes? ¿quiénes son los usuarios de la biblioteca y de qué tipo de materiales y servicios hay que disponer para hacer frente a sus necesidades? ¿qué relaciones cooperativas hay que establecer con otras bibliotecas y otros proveedores de información?

La creación de unas normas de desarrollo de la colección sirve para diversas finalidades:

a) Como una herramienta de planificación para determinar el uso de los recursos monetarios y de personal.

b) Como una guía de ayuda para que los encargados de la selección desarrollen las colecciones.

c) Como un medio de asegurar que las colecciones se desarrollan de manera consistente a través del tiempo y de modo que los cambios de personal le afecten en la menor medida posible.

d) Como una vía de entrenamiento para los nuevos profesionales bibliotecarios.

e) Como establecimiento de una filosofía de actuación.

f) Como defensa respecto a ataques referidos a la calidad y cantidad de materiales que integran la colección.

Ha de ser, obviamente, la sección o departamento de gestión de la colección de materiales de la BCC quien adapte estos criterios generales, aplicables a cualquier biblioteca, a las especiales características de los centros objeto de nuestra atención.

De los diversos objetivos que Buckland y Hindle (15) señalan, con carácter general, como aplicables a la gestión de la colección de materiales de un centro (seis, en concreto), aquí vamos a referimos a algunos de ellos especialmente aplicables a las BCC:

1. Amplitud, que no necesariamente se refiere al crecimiento cuantitativo y acrítico de la colección, sino que puede referirse a que abarque toda la literatura de calidad. Ello no obstante, resulta más que evidente que cuando usamos esta idea de «amplitud», en la mayoría de las bibliotecas se piensa en el crecimiento cuantitativo, partiendo del conocido (y posiblemente erróneo) criterio de «cuanto más, mejor».

Aplicado a la colección de materiales de las BCC, el criterio de amplitud es de esencial importancia, pues nos movemos entre el objetivo ideal de incorporar a ella cualquier ítem que se refiera a la Comunidad en cuestión y la realidad de lo imposible de ese deseo, ya sea por meras carencias presupuestarias o, simplemente, porque ello requeriría tal cantidad de recursos humanos que lo haría fácticamente irrealizable.

La cuestión siempre espinosa, y más aún en centros de las especiales ca- 
Las bibliotecas centrales de Comunidades Autónomas del Estado Español. Su colección de materiales

racterísticas de los que estamos estudiando, de los criterios para la selección de materiales que hay $\longrightarrow$ no- que incorporar a la colección de materiales, se transforma en algo vital, si no queremos acabar organizando monstruos inmanejables en los que meros e indiscriminados criterios cuantitativos de crecimiento hagan difícil, por no decir que casi imposible, el trabajo con una colección en la que la excesiva cantidad haya ahogado unos mínimos criterios de calidad. Este problema resulta agravado porque, al tener las Bibliotecas Centrales de Comunidad Autónoma el carácter de centros donde se debe conservar - al menos esa es la teoría de la que se parte - toda la producción documentaria de su Comunidad, se produce un más que evidente conflicto entre la utopía de conservarlo todo y la realidad de que, en más ocasiones de las que pueda pensarse, ello resulte ciertamente imposible, aún cuando esos materiales se refieran a un área geográfica de suyo no excesivamente amplia. Disponibilidad. El concepto de disponibilidad fue inventado para referirse a la diferencia entre teoria y práctica respecto a la colección considerada en su globalidad, cuando todos los títulos requeridos están teóricamente disponibles (sobre todo, teniendo en cuenta la aportación esencial de las redes telemáticas) pero, en la práctica, ello resulta obviamente imposible.

$\mathrm{Si}$ bien es cierto que Internet nos puede proporcionar prácticamente cualquier material que necesitemos para la colección, no lo es menos que esa misma superabundancia genera una esencial dificultad para encontrar lo que realmente deseamos, máxime en temas de suyo tan restringidos como puedan ser los que atañen a una biblioteca como las que, aquí, son el objeto de nuestra atención.

\subsection{Criterios de planificación estratégica de la colección}

Como criterios generales de esa planificación estratégica aplicada a la colección de materiales de las BCC podríamos señalar, entre otros, y adaptándolos a las especiales características de nuestros centros, los establecidos por la Biblioteca Nacional de Australia (16); son los siguientes:

1. Aumentar en el mayor grado posible el alcance y uso de la información basada en entornos electrónicos.

2. Destacar el esencial carácter de estos centros como proveedores de recursos de información y de servicios para la investigación en todo lo que se refiere al patrimonio bibliográfico y documentario de la Comunidad en cuestión.

3. Desarrollar un activo programa para coleccionar y conservar cualquier tipo de material documentario que se refiera a la Comunidad.

4. Asegurarse de que la BCC es el centro principal y puntero en la recogida de materiales, tanto en formato papel como electrónico, en cuanto a cualquier tema que se refiera a su Comunidad. Esto habrá de llevarse a cabo mediante la identificación y evaluación de nuevas fuentes de información en cualquier formato y mediante el desarrollo continuo de un activo programa de adquisiciones que los haga disponibles; ello supone tener muy en cuenta los costes, el almacenamiento, la preservación, la accesibilidad y las preferencias 
de los usuarios al establecer las colecciones, los recursos de información y las necesidades de equipamiento.

5. Asegurarse de que las normas para el desarrollo de la colección son especialmente sensibles a los temas de importancia básica en cada Comunidad (los vinos, en la Rioja; la moda, en Galicia; la agricultura hortícola y frutera en Murcia, etc.) de modo que dichas áreas temáticas constituyan auténticos puntos focales de atención.

6. Apoyar, mediante el desarrollo de la colección, los aspectos nuevos y punteros de la Comunidad en lo que se refiere a sus principales y emergentes líneas de estrategia económica, social, etc.

7. Asegurar que la política de desarrollo de la colección incorpora los tópicos relativos a las minorías raciales, culturales, etc. (Los vaqueiros de alzada en Asturias, los chuetas mallorquines, los gitanos - de quiénes resulta obvio señalar su enorme y continua aportación a la cultura española-, establecidos en prácticamente toda España, etc.), de modo que la colección refleje la diversidad cultural y vital de la Comunidad en cuestión.

8. Participar, junto con otras instituciones culturales del área geográfica específica (grandes bibliotecas públicas, sistema de bibliotecas universitarias, etc.) en el establecimiento de estrategias cooperativas para el desarrollo de la colección, de modo que ello refleje un creciente interés más en la accesibilidad a los materiales que en su mera posesión.

9. Desarrollar un sistema cooperativo que haga posible las más fácil accesibilidad de las bibliotecas públicas de la zona, con independencia total de su ubicación geográfica.

10. Desarrollar un sistema de cooperación electrónica con los principales sistemas de información de la Comunidad, especialmente con los que se refieren a las bibliotecas públicas y universitarias, sin excluir las más importantes bibliotecas especializadas.

11. Contribuir al desarrollo de esquemas cooperativos que permitan compartir experiencias y racionalizar el desarrollo de las colecciones, muy especialmente en lo que se refiere al patrimonio bibliográfico.

\subsection{Desarrollo de la planificación estratégica de la colección}

Como elemental punto de partida habría que señalar que la planificación no ha de ser, en modo alguno, la labor de un director de bibliotecas actuando aisladamente, ni siquiera si lo hace contando con su equipo interno de colaboradores del centro; el grupo de trabajo que para ello se constituya, durante un período mínimo de seis meses, en los cuales tendrán varias reuniones, habrá de contar con la colaboración e implicación directa de las más importantes bibliotecas de su Comunidad; ese grupo redactará un borrador que servirá como punto de partida para el debate y discusión de los diversos grupos que posteriormente se constituirán y que tendrán una base temática, según las variadas materias y formatos que van a ser los núcleos fundamentales de la colección; lejos ya los tiempos en que un más o menos inspirado y afortunado director de la BCC era el único que establecía los criterios básicos para la constitución de la colección. 
Estos grupos de trabajo, a los que podríamos denominar «grupos temáticos», desarrollarán los criterios referentes a materias y formatos específicos en los que debe incidir especialmente la colección y contarán entre ellos a cualificadas bibliotecarias y bibliotecarios de bibliotecas públicas, pero sin obviar la presencia en ellos de otras y otros procedentes de las más importantes bibliotecas universitarias y especializadas de la Comunidad.

En la elaboración de este documento base para el desarrollo de la colección de la Biblioteca Central de la Comunidad también habría que contar con la participación de las asociaciones de bibliotecarias y bibliotecarios de la Comunidad y, si existen, con los centros universitarios que se dedican a la formación de los futuros profesionales del sector.

Si en una primera fase un grupo ha elaborado un documento de carácter generalista, que luego ha sido completado por los grupos temáticos a los que hemos hecho mención, en una tercera fase, y como consecuencia lógica de las dos anteriores, aparece un documento, ya más profundo y elaborado, que debe circular entre todos los profesionales de este sector en la Comunidad que quieran implicarse en ello; en esta fase, estos profesionales harán cuantas sugerencias estimen oportunas al documento que se les presenta, de modo que el documento final resultante constituirá una verdadera normativa para el más eficaz y eficiente desarrollo de la colección.

El cumplimiento acertado de cada una de estas fases nos proporcionará un documento final que, al menos en teoría, debe responder a los diversos puntos de vista de los profesionales bibliotecarios de la Comunidad, resultando de ello no sólo lo que en ese momento parece más factible, sino que, al contar con toda una gama diversa de profesionales bibliotecarios que han participado en su elaboración, de modo que sólo se haya excluido a quien no haya deseado participar, dará como consecuencia colateral y muy positiva el que todas las bibliotecarias y bibliotecarios de la Comunidad se sientan realmente relacionados con la BCC, con sus problemas y con las soluciones pertinentes.

No obstante lo expuesto respecto a la formación de los grupos que deberían encargarse de la planificación de criterios para constituir y desarrollar la colección de materiales, autores hay que, como Dowlin (17), se muestran en desacuerdo con este proceso «tradicional», podríamos decir, de formación de dichos grupos de trabajo, afirmando al respecto: «Más que la selección de títulos mediante el proceso tradicional centrado en un comité, la decisión debería conllevar la selección de vendedores y editores y a partir de ahí tener la información publicada por ellos en cada año. Este enfoque proporcionaría muchas posibilidades de negociación y eliminaría grandes cantidades de trabajo innecesario».

\subsection{Evolución, control y evaluación del plan estratégico del desarrollo de la colección}

Incidimos, en primer lugar, en la idea de que nos estamos refiriendo a la evolución, control y evaluación del plan estratégico en sí mismo considerado y no a dichas cuestiones aplicadas a la colección propiamente dicha; es decir, analizamos la estructura de acción, no aquel elemento sobre el que dicha estructura va a desarrollar su trabajo. 
Resulta evidente que el desarrollo de las normas que se hayan propuesto debe estar sujeto a constantes y continuos ajustes, pues inexorablemente se van a producir cambios en los niveles presupuestarios de que se parta (ya sean cambios positivos, al alza, o negativos, lo más probable, en las cuantías presupuestarias con que teóricamente se cuenta en las fases de iniciación).

Junto a los cambios de raíz económica, presupuestaria, están los cambios en el nivel organizativo, en las estructuras de trabajo y, unidos a ellos, los cambios en los materiales propiamente dichos, cambios éstos a la orden del día, ya sea por la aparición de nuevos formatos, por el influjo de la información que llega por vía telemática, etc.

Además de los cambios que proceden de las circunstancias económicas, de la esfera organizativa y de los referentes a los materiales en sí mismos considerados, hay que referirse a aquellos otros que tienen su raíz en los usuarios, en sus también mutables necesidades de servicios, en lo que realmente esperan obtener de las BCC.

$\mathrm{Si}$ en un principio el usuario tipo, modelo podríamos decir, de las BCC es el investigador, el profesor, el erudito, etc., esta tipología está cambiando a marchas forzadas, de modo que las BCC deben estar al tanto de una nueva tipología de usuarios mucho más diversa y, por tanto, con un abanico de necesidades y deseos muchos más multiforme y heterogéneo.

Para hacer frente a todas estas nuevas circunstancias las BCC deben adoptar una actitud no meramente reactiva, sino esencialmente proactiva, de modo que sean capaces de captar los nuevos aires y tendencias que se van a producir para, con los datos necesarios, ser capaces de hacerles frente de manera eficaz y eficiente.

Todo ello implica, necesariamente, que la planificación estratégica básica de la que hayamos partido al elaborar las normas de desarrollo de la colección debe estar continuamente sometida a control y evaluación; desde este enfoque considerada, la planificación de la colección, al igual que cualquier otro tipo de planificación, ha de ser dinámica; en un mundo como éste, tan sometido a constantes cambios de todas clases, de nada serviría una planificación que, por mucho tiempo que haya tardado en ser puesta en marcha, no sea capaz, asimismo, de saber adaptarse a las nuevas circunstancias que van a ir apareciendo.

De todos estos cambios señalados, los dos más importantes, con diferencia, son los que se refieren a las nuevas vías de acceso a la información y, junto a éstos, los que atañen a los usuarios.

A estas alturas resulta ya un tópico, inevitable por otra parte, referirse a la revolución en los modos de acceso a la información, pero hay que destacarlo siempre pues, si las BCC no se suben ya al carro de las nuevas posibilidades que aparecen desde la perspectiva tecnológica, acabarán siendo, no más, especies en extinción, recordables en la historia del devenir de las bibliotecas, ciertamente, pero escasamente útiles para lo que las actuales circunstancias requieren.

Este planteamiento del acceso a las nuevas tecnologías en cualquier biblioteca actual, sea del tipo que sea, no es sólo, cómo se comprenderá, el adecuado para adaptarse a lo que los tiempos actuales requieren, sino que lleva implícito todo un nuevo enfoque de la cuestión desde una perspectiva netamente política; de este modo, como ejemplo - uno entre mil- del nuevo análisis de la cuestión, pueden servirnos las palabras del premier británico Tony Blair cuando dice: «Las autopistas de la información no deberían beneficiar sólo a los pudientes o a los habitantes de la ciudad. De la misma manera que en el pasado los libros supusieron una oportunidad para que la gen- 
te corriente se mejorara a sí misma, en el futuro la educación en línea será un camino para las nuevas expectativas. Pero de la misma manera que los libros están disponibles en las bibliotecas, los beneficios de las autopistas de la información deben estar allí al alcance de todos. Esto supone una posibilidad real en la igualdad de oportunidades» (18).

Unido a esto, el atender al nuevo tipo de usuario que surge cuando la información ya no es la meramente presencial en el centro y mediante el soporte gutenbergiano, el viejo y amado papel cuya vida Dios guarde para siempre; ahora, las BCC deberán saber - lo saben ya-, que la colección no sólo es real, en papel, también es virtual, está en la Red, se puede comprar su posesión real o sólo el acceso a ella, y eso implica el replantearse totalmente qué servicios están en disposición de ofrecer y para qué tipo de usuarios en concreto.

Conclusión de lo que estamos diciendo es la elaboración de un adecuado programa de evaluación de ese plan estratégico de desarrollo de la colección, de modo que esta evaluación sea inicial, desde el comienzo del trabajo de planificación, cuando estamos diseñando las bases teóricas que van a ser su soporte y guía de acción, sea también evaluación de cómo está funcionando el programa de planificación, para ver si sigue o se separa - y en qué medida - de las líneas de actuación diseñadas y, concluyendo, ha de ser también una evaluación finalista, continua; en tanto se quiera tener una buena planificación del desarrollo de la colección ello requiere necesariamente una evaluación periódica que haga frente a las circunstancias nuevas que se van a ir produciendo.

Planificar, como es sabido, no es dar unas reglas que nos van a servir para siempre; es señalar unas vías de acción lo suficientemente ágiles y flexibles que nos van a servir como asidero para poder resolver nuevos problemas; si la colección es algo vivo, que crece, que cambia atendiendo a nuevas necesidades, su planificación no puede en modo alguno ser una vía de acción inmutable. La mejor, si no acaso la única, manera de contar con la planificación más adecuada, es una evaluación de ella(19).

\section{Los materiales}

La primera cuestión, de tipo puramente práctico, a la que deben atender las BCC se refiere a la enorme y diversa tipología de materiales que, por la propia naturaleza de estos centros, deben recoger; si la diversa tipología a la que hacemos referencia entra dentro del orden de lo cualitativo, otro tanto podemos decir en cuanto al aspecto cuantitativo de la colección, pues un atento seguimiento de la siempre problemática cuestión del Depósito Legal (y posteriormente volveremos, por su importancia excepcional, a tratar de este tema) conlleva un aumento a veces exponencial de los materiales que se recogen.

En cuanto a los diversos materiales que recaban para sí la atención de las BCC citamos, como cosa conocida, los libros y publicaciones periódicas, los manuscritos, los libros raros y antiguos, los mapas, las microformas, la documentación musical, los periódicos, los materiales efímeros, las publicaciones oficiales, los soportes de historia oral y folklore y, finalmente, por su excepcional importancia hoy día, las publicaciones electrónicas.

De esta amplia relación de materiales en la que, cierto es, se combinan criterios 
referidos tanto al soporte en sí mismo (libros) como a su contenido específico (publicaciones oficiales), pero que hemos mantenido como la más práctica siguiendo los criterios clasificatorios más aceptados en estas materias, vamos a comentar, siquiera brevemente, aquéllos que, por así decir, no suelen ser los más comunes o que plantean problemas concretos por su especificidad.

Cierto es que, dada la rapidez con que la tecnología ha revolucionado el almacenamiento, recuperación y entrega de información en las últimas dos décadas, la cuestión respecto al formato definitivo de los materiales almacenados debiera ser superflua; no obstante, no es menos cierto que de momento, y hasta no sabemos cuándo, los soportes «tradicionales» van a seguir existiendo, de donde la justificación de dedicarles una amplia atención.

\subsection{Manuscritos, libros raros y antiguos}

Los manuscritos, libros raros y antiguos no suelen constituir un bloque muy importante de materiales en las BCC, y ello es así por la lógica razón de que son bibliotecas de muy reciente creación. Referidos al caso español, este tipo de materiales constituye colecciones de excepcional importancia en las bibliotecas de corte tradicional (Biblioteca Nacional de España, bibliotecas provinciales - donde, cómo es sabido, el grueso de estas colecciones procede de la Desamortización de Mendizábal en el siglo XIX-, bibliotecas universitarias más antiguas, etc.) (20).

En el caso de las BCC resulta casi imposible, por los altísimos precios de estos materiales y por la dificultad de encontrarlos para su compra, el que estos centros lleguen a poseer una colección medianamente interesante lo que, por otra parte, tampoco supone ningún problema especial, y ello por dos razones:

1. Al existir en todas las Comunidades Autónomas buenas colecciones de estos materiales, lo que sí ha de proporcionar cada BCC es la información global de los materiales existentes en su área geográfica y, en cuanto a esta cuestión, ello se consigue adecuadamente mediante programas como el Catálogo Colectivo del Patrimonio Histórico Bibliográfico que, centralizado en Madrid y en el Ministerio de Educación y Cultura, proporciona dicha información, como es bien sabido, a través de la base de datos PABI (21).

2. Un adecuado programa de copia, ya sea de microfilmación o digitalización, permite tener en cada BCC aquellos ejemplares que se consideren imprescindibles; esto, que en principio podría parecer un gasto superfluo, puede resultar en la práctica enormemente beneficioso para los investigadores de la Comunidad, ya que, en un primer acercamiento a la obra que necesitan, se les ahorra el tener que desplazarse para consultar los ejemplares deseados; únase a esto el hecho evidente de que la digitalización normalmente conlleva la puesta en red de los materiales digitalizados. Lo ideal sería que esta labor de duplicación no se lleve a cabo de manera aislada y en casos concretos y específicos, sino obedeciendo a un seriamente planificado programa de actuación en el que se hayan estudiado todos los factores que inciden en la cuestión, desde la importancia real de las materiales que van a ser duplicados a la valoración del programa, sin olvidar temas de suyo tan importantes como los recursos humanos implicados, el presupuesto, las posteriores condiciones de ubicación y conservación de estos materiales, etc. 


\subsection{Mapas}

Bajo la denominación general de «mapas» recogemos, obviamente, todos los materiales que resultan englobados bajo el denominador común de «materiales cartográficos». En la mayoría de BCC estas pueden, y deben, convertirse en el principal referente de su Comunidad respecto a este tipo de materiales.

Conseguir una buena colección de materiales cartográficos requiere una adecuada planificación de partida, en la que se tengan en cuenta cuestiones tales como el presupuesto que se va a destinar, los proveedores, los recursos humanos.

Conviene no olvidar que las especiales características de tamaño y formato de estos materiales hace imprescindible un muy serio estudio de la ubicación que se les va a dar, de los elementos de mobiliario adecuados y necesarios (armarios de amplios cajones horizontales, huyendo de los de tipo vertical en que planos y mapas aparecen "colgados» y que tan difíciles resultan de manejar); otro tanto hay que decir de la funesta costumbre, practicada en algunos centros, de conservar estos materiales enrollados en tubos de cartón, lo que supone que el tiempo se encargará de que, antes o después, sea imposible desenrollarlos, pues habrán cogido tal grado de curvatura que, nada más abrirlos, se cuartean.

A la hora de proceder a la adquisición de estos materiales se puede acudir a un proveedor normal, ya sea una editorial, una distribuidora o una librería, pero resulta adecuado tener en cuenta que comprar directamente a los grandes centros que en España los producen resulta eficaz y eficiente, es decir, proporcionan la mejor cartografía y al mejor precio.

Los principales centros que en nuestro país, como es bien sabido, producen materiales cartográficos (y la relación que de ellos presentamos en modo alguno pretende ser exhaustiva, sino meramente orientadora) son el Instituto Geográfico Nacional (cuya cartografía puede ser adquirida a través del Centro Nacional de Información Geográfica (22)); el Servicio Geográfico del Ejército (23) y el Instituto Hidrográfico de la Marina (24).

Junto con estos centros de carácter estatal, conviene no olvidar que algunas Comunidades Autónomas poseen centros similares. Además, a la hora de organizar una completa sección de materiales cartográficos, tanto las Diputaciones Provinciales como los ayuntamientos más importantes tienen áreas dedicadas a la elaboración de planos y mapas, de mayor o menor alcance, respecto a su territorio.

$\mathrm{Si}$ hasta aquí nos hemos referido a la cartografía «tradicional», es preciso hacer mención de la cartografía digital, de excepcional importancia para los investigadores. Aunque su precio es muy superior al de los otros materiales, hoy día resulta imprescindible en una buena sección de cartografía.

\subsection{Microformas}

De entrada hay que decir que, aunque parezca que vamos a contracorriente e ignorando los nuevos soportes de transferencia de información, pensamos que los micromateriales todavía pueden ejercer un más que útil papel en los centros objeto de nuestra atención. Hasta ahora han demostrado tener, con el tratamiento adecuado en cuanto a las circunstancias medioambientales y de seguridad para su mantenimiento y 
conservación, unas aceptables condiciones; únase a ello que su precio, comparado con el de los nuevos soportes digitales (de los que, por cierto, casi nada sabemos sobre cuánto van a durar) es netamente inferior, no tanto en lo tocante al soporte en sí mismo considerado sino, más aún, en cuanto a los aparatos (hardware) que requieren, de mucha menos complicación y precio que para los materiales digitales.

No estamos defendiendo absolutamente, quede bien claro, a unos materiales sobre los otros; simplemente afirmamos que, hoy por hoy, los micromateriales pueden seguir siendo, en muchos casos, realmente útiles.

Conviene no olvidar, no obstante, que ni hay ni debe haber ninguna oposición entre el hecho de microfilmar y la digitalización, de modo que en los más importantes centros, si las condiciones económicas lo permiten, la microfilmación suele ser un primer paso en la digitalización.

La sección de micromateriales, como ya decíamos al hablar de los manuscritos, puede servirnos muy eficazmente para poder tener en la colección de materiales todos aquellos elementos a los que de otra manera nunca podría acceder la BCC. En cualquier caso, la selección de estos materiales obliga a usar los mismo criterios restrictivos y de valoración utilizados cuando se trata de materiales en formato tradicional.

Normalmente, y sin prescindir de otros, el uso más general de los micromateriales ha sido, junto con la adquisición de obras en formato original de manuscrito, raro, etc., el referente a las publicaciones seriadas y periódicas, de modo que mediante esta sección cualquier BCC puede contar con los más importantes títulos referidos a su Comunidad.

El principal problema que plantea esta sección, como ya hemos dicho, es el de su conservación, dependiendo ello en gran manera de las condiciones de temperatura y humedad que sufran estos materiales.

\subsection{Materiales efímeros}

Englobamos en estas dos palabras lo que las bibliotecas del mundo anglosajón recogen bajo la etiqueta de «ephemera». Nos referimos, cierto es, a todos esos materiales (folletos de propaganda, programas de mano, carteles, etc.) que, en la mayoría de nuestros centros reciben poca, por no decir ninguna, consideración. No se vea ninguna crítica en lo que decimos: sobrepasado totalmente el personal por la avalancha de libros, publicaciones periódicas, etc., que se reciben en los centros, y unido ello a una casi sempiterna situación de falta de los recursos humanos necesarios o adecuados, resulta comprensible que, en el mejor de los casos, el único tratamiento que reciben estos materiales no vaya más allá del almacenamiento.

Son, por así decirlo, los «testigos menores» de la historia; hoy no tienen prácticamente ningún valor, pero el paso del tiempo irá poniendo sobre ellos, sin duda, esa pátina de respeto, de veneración casi, que nos sugieren elementos tales como una entrada para una corrida de toros de hace un siglo, o el menú de fin de año de una sociedad pública hace cincuenta años, o la invitación personal a tal o cual ceremonia de una localidad hace de ello décadas ya, etc.

Tal vez el error, a la hora de organizar y conservar estos materiales, esté en pretender someterlos al mismo procedimiento de descripción formal y de contenido que si se tratase de una monografía o un artículo de revista. Obviamente, no es ese el caso; hay que arbitrar nuevos procedimientos, entendiendo que las Reglas de Catalogación 
y demás torturantes herramientas no son, en sí mismas consideradas, más que un medio, y nunca un fin, como a veces parece que se da a entender.

Si para algo deben servirnos las nuevas tecnologías (y he aquí posiblemente, y esta de mucho mayor gravedad, otra confusión entre medios y fines) es para liberarnos de los trabajos puramente rutinarios y para que los bibliotecarios se dediquen en cuerpo y alma a aquello que debe ser su objetivo primordial: atender a sus usuarios.

\subsection{Historia oral y folklore}

Por tradición o por la desconocida razón que sea, y a diferencia de lo que sucede en otros ámbitos culturales distintos al nuestro, la cultura oral no ha recibido en nuestros centros demasiada atención; ninguna, o casi, por mejor decir. No se trata de que, de la noche a la mañana, los bibliotecarios se transformen en antropólogos o historiadores de la cultura oral, pero creemos sinceramente que si hay un centro que debiera ocuparse de recoger todo el testimonio vivo de las generaciones, y ello desde que existen los medios materiales y técnicos para poder hacerlo, ese centro podría ser la BCC, dado su carácter de almacén de todo un patrimonio cultural. Aquí, así lo entendemos, habría que procurar salir del encorsetamiento a que conduce el pensar que la biblioteca es el lugar casi exclusivo de lo publicado, ya sea en papel o en cualquiera de los múltiples soportes nuevos o por venir.

La cultura oral, tan rica en un país como España, se pierde día a día porque los nuevos modos del vivir hacen que otras maneras de entender la vida, mejores o peores pues ello no viene al caso, estén desapareciendo. El ejemplo de otros países está en que el centro que, de suyo, se dedica a recoger y conservar estos materiales es la biblioteca y, en nuestro caso, ninguna mejor situada para llevar a cabo esta labor que la BCC.

Claro es que supone un nuevo planteamiento en cuanto a la planificación de esta actuación y a los recursos humanos y materiales que a esta labor habría que dedicar, pero pensamos que se abriría toda una vía de trabajo que podría conseguir incardinar más y mejor a la BCC con la sociedad a la que tiene que servir.

Reseñemos, para acabar este epígrafe, que si bien desde el punto de vista español (y en lo que se refiere al enfoque bibliotecario de esta cuestión) la bibliografía existente es mínima, por no decir nula, ésta es bien fácil de encontrar en otras lenguas y países, para aquellos profesionales que quisiesen avanzar en este campo del conocimiento que, al fin y al cabo, no supone sino conservar, aunque en otro formato y con otros métodos, los valores culturales y la manera de entender la vida de unas generaciones que están desapareciendo.

Si las BCC recogen, o lo intentan, todo lo publicado referente a su Comunidad, en una amplia gama que va desde el tradicional libro a las novedosas publicaciones electrónicas, no vemos razón objetiva alguna para que no dediquen parte de sus esfuerzos a recoger estos materiales, de suyo tan importantes para un auténtico conocimiento de su propia realidad.

\subsection{Publicaciones electrónicas}

Pese a la enorme cantidad de cuestiones y problemas que el uso de materiales electrónicos y digitales presenta en cualquier biblioteca actual, y en no menor medida 
- tal vez todo lo contrario - en las BCC, no vamos a entrar en este artículo, de carácter general, en la inmensa variedad de enfoques que precisa este tema cuando lo tratamos desde el punto de vista de su incorporación como materiales de la colección. Cuestiones como la diversidad de soportes, el acceso a o posesión de los materiales y de la información que conllevan, los criterios de selección, la planificación general de su uso y tratamiento, las necesidades cambiantes de los usuarios, etc., todo ello configura un extenso abanico de temas que merecen tratamiento específico y aparte.

\section{Depósito Legal y colección de materiales}

De entre las diversas fuentes de aprovisionamiento para la colección de materiales (adquisición, intercambio, donativos), el Depósito Legal es, para las BCC, una de las más importantes, y así queda establecido, sin excepción, en la normativa por la que estos centros se rigen.

No vamos a plantear los más que numerosos problemas que esto supone, en cuanto a legislación, control y proceso de recogida de materiales, etc. (25), sino que nos centramos en los problemas nuevos que aparecen cuando una de las crecientes vías de incorporación de materiales viene dada por el uso de las redes telemáticas y, más en concreto, por la posibilidad de incorporar al fondo de la biblioteca materiales obtenidos a partir de Internet (26).

La primera cuestion que surge viene dada, paradójicamente, por el hecho de la superabundancia de materiales a los que se puede acceder, de modo que la existencia de unos claros criterios para la selección e incorporación de estos materiales a la colección resulta, más que necesaria, absolutamente imprescindible; una elemental sensatez en esta cuestión indica que en todos los casos deben prevalecer criterios de calidad frente a los meramente cuantitativos (27).

Para Navelsaker (28), los principales criterios aplicables a la recogida de materiales electrónicos mediante el Depósito Legal son los siguientes:

- Que los documentos estén disponibles al público general y que su formato original sea electrónico.

- Que los documentos puedan ser considerados de un valor permanente para la comprensión de la sociedad actual.

Según estos criterios, Mc Gowan (29) establece que las Bibliotecas Nacionales (y las BCC, obviamente: el paréntesis es nuestro) deben recoger materiales tales como:

a) Publicaciones electrónicas que «imitan» a las publicaciones tradicionales (libros, periódicos, revistas).

b) Literatura interactiva.

c) Publicaciones multimedia.

d) Programas educativos de ordenador.

e) Grupos nacionales de noticias de la red; en nuestro caso, se trataría de grupos de noticias existentes en la Comunidad Autónoma pertinente.

f) Grupos de debate existentes en las listas de servidores.

g) Bases de datos seleccionadas 
En torno a esta misma cuestión, Wille (30) propone los siguientes criterios para el Depósito Legal de materiales electrónicos:

a) El origen nacional de las publicaciones.

b) La audiencia a la que va destinada la publicación.

c) La importancia para el patrimonio social y cultural del país.

d) Su significación e importancia para futuras investigaciones.

Fagerli, al analizar el complejo problema del manejo de documentos electrónicos en el contexto del Depósito Legal, expone cómo ha cambiado su propio pensamiento desde el modelo de una Biblioteca Nacional (o, en nuestro caso, de una BCC) que proporciona el acceso tradicional en línea hacia una variedad de bases de datos desde una «sala electrónica de lectura».

Esta solución que sugiere refleja las preferencia de los profesionales de la información automatizada por los sistemas distribuidos y en red sobre los almacenes de datos centralizados, ya que el rápido desarrollo de la Red proporciona un mecanismo efectivo para el control nacional de la información dinámica.

Concluye Fagerli afirmando que:

a) El Depósito Legal de los documentos dinámicos debe ser completado por las Bibliotecas Nacionales (o BCC, en nuestro caso) mediante el acceso a los documentos.

b) Los instrumentos de acceso deben estar constituidos como páginas de entrada (home page) internacionales y normalizadas. Esta «página de entrada» debe hacer las funciones de archivo bibliográfico nacional de estos documentos(31).

\section{Conservación}

No es éste el lugar donde exponer en profundidad el complejo cúmulo de cuestiones que un tema como éste de la conservación lleva anejo; nos limitamos a señalar que un buen programa de gestión de la colección resulta incompleto si en él falta el establecimiento de un plan de acción en estas materias. Si partimos de que la conservación de materiales es una de las características, por su propia naturaleza, de las BCC, se comprenderá fácilmente la evidencia de lo que estamos señalando.

Como mejor vía operativa, conviene pensar en la conservación tanto desde el inicio como durante el desarrollo de la planificación general de la colección.

Esto implica:

- La valoración, en cantidad y calidad, de los diferentes soportes que van a ser incluidos en la colección.

- El tomar en consideración las características estructurales y espaciales del edificio.

Sus condicionantes medioambientales y niveles de seguridad, no sólo en lo que se refiere a la precaución ante procesos naturales (normales o excepcionales) que puedan dañar a los materiales, sino también en lo que respecta a la seguridad ante daños por vandalismo, etc. 


\section{Expurgo}

Parecerá una incongruencia que, si estamos hablando de un tipo de bibliotecas, las BCC, que tienen una especial misión en cuanto a la conservación de materiales, comentemos el tema del expurgo, pero lo hacemos no desde una perspectiva teórica, sino basados en experiencias prácticas.

Como en cualquier otro tipo de biblioteca, en las BCC se acaba conservando toda una serie de materiales que, bien por ser duplicados, bien por no corresponder a las líneas generales de formación de la colección, etc., acaban no siendo más que un peso muerto, un lastre.

Si la planificación de una acción, continua y constante siempre, de expurgo ya es de suyo harto agobiante en cualquier tipo de biblioteca, la cuestión se complica enormemente en las bibliotecas objeto de nuestra atención, pero no por ello ha de ser soslayado este espinoso tema.

Sírvanos, de momento, su mero planteamiento como una más de las actividades a las que hay que hacer frente cuando planificamos el desarrollo de la colección

\section{Conclusión}

Podríamos resumir brevemente lo hasta aquí expuesto diciendo que si las Bibliotecas Centrales de Comunidades Autónomas saben hacer frente a los retos que plantea la sociedad de la información, definiendo adecuadamente cuáles son las misiones y tareas que les corresponden en un panorama, el de la información, que cambia día a día y adaptándose adecuadamente a esos cambios, de modo que su colección de materiales, tanto en los elementos que la constituyen como en cuanto a su gestión, sea una auténtica herramienta de adaptación a los nuevos soportes y vías de acceso a las nuevas tecnologías, tienen evidentemente un más que envidiable futuro.

Adaptarse inteligentemente a estos cambios supone convertir las debilidades en fortalezas y transformar las amenazas en oportunidades.

Así sea.

\section{Bibliografía}

1. LINE, M. B. National Libraries. London: ASLIB, 1979.

2. GOODRUM, C. A. National Libraries. En: ALA World Encyclopedia of Library and Information Revue. Chicago: ALA, 1980.

3. WILSON, A. Problems in determining the role and function of the national library as part of the library and information service development. En : DUDLEY, E. (editor): The development of National Library and Information Services. Papers given at the First Library Association International Workshop. London.1981. London: The Library Association, 1983.

4. En: Biblioteca de Aragón. Marco Jurídico. http://www.aragob.es./bibara/pagiba/legiba.htm.

5. En lo relacionado con la legislación bibliotecaria de las Comunidades Autónomas de España resulta de interés la consulta de: http://www.fundacióngsr.es/servicios/frames.htm.

6. MORHOLT, P. Libraries as bridges. Librarians as builders. En: LANCASTER, F. W. Li- 
braries and the future. Esssays on the library in the twenty-first century. New York, etc.: The Haworth Press, 1993 p. 27.

7. GORMAN, M. Five new laws on librarianship. En: American Libraries, 26(8), 784-785.

8. McGOWAN, Ian. National Libraries. En: Librarianship and Information World, 1996/1997. An annual survey. General Editor Maurice B. Line. London, etc.: Bowker, 1997.

9. LINE, M. B. Back to basis for National Libraries? En: Alexandria, 7(1), 1-2 (editorial).

10. BETTEN, L. IFLA Konferenz 1995, Estambul, Buch und Bibliothek, 47 (11/12), 10341037

11. McLUHAN, M. La galaxia Gutemberg: Génesis del «homo typographicus». Barcelona: Círculo de Lectores, 1993.

12. BROWN, G. The management of knowledge: Libraries. En: American Libraries, 26(3), 222-224.

13. KOONTZ, H; WEIHRICH, H. Administración. Una perspectiva global. 10." ed., México: McGraw Hill, 1994, p.118.

14. RUBIN, R. E. Foundations of library and information science. New York: Neal-Schuman, 1998, p. 140.

15. BUCKLAND, M. K.; HINDLE, A. Acquisitions, growth and performance control through systems analysis, en GORE, D.: Farewell to Alexandria: Solutions to space, growth and performance ploblems of libraries. Westport (Co.): Grenwood Press, 1976, p. 44-61.

16. STATE Library of South Australia Strategic Directions. - En: http://www.slsa.sa.gov.au/collres/collres/future.htm.

17. DOWLIN, K. E. The neographic library. A 30 - year perpective on public libraries. En: LANCASTER, F. W. Libraries and the future. Essays on the library in the twenty-first century. New York, etc.: The Haworth Press, 1993, p. 34.

18. BLAIR, T. New Britain. My vision of a young countrry. En: http//:www.ukoln.ac.uk/services/lic/newlibrary/introduction/html.

19. FUENTES ROMERO, J. J. Evaluación de bibliotecas y de servicios de documentación $e$ información. Gijón (Asturias): Trea, 1999.

20. Es de justicia reseñar que existen notables excepciones en cuanto a las colecciones de manuscritos, raros, etc., de alguna que otra Biblioteca Central de Comunidad Autónoma; sirva como ejemplo el caso de la novísima, por sus instalaciones, y ejemplar Biblioteca Valenciana

21. PABI: http://www.mcu.es/ccpb/index.html.

22. Centro Nacional de Información Geográfica: http://www.cnig.es/cimpr.htm.

23. Servicio Geográfico del Ejército. Publicaciones en : http://www.mde.es/mde/public/publi$\mathrm{ca}$ pdf/alfabetico.pdf.

24. Instituto Geográfico de la Marina: http://www.armada.mde.es/ihm/espa/catalog.htm

25. Para ahondar en estas cuestiones resulta enormemente provechosa la monografía del profesor José Antonio Cordón García: El registro de la memoria. Las Bibliografías Nacionales y el Depósito Legal. Gijón (Asturias): Trea, 1997.

26. Sobre este tema del Depósito Legal y el Copyright de los materiales existentes en la Red o en formato electrónico fuera de ella resulta interesante consultar el informe que el Deparment for Culture, Media and Sport del Reino Unido encargó a la National Grid for Learning; este organismo contituyó la Library and Information Comission, que elaboró el informe New Library. The People's Network; este informe supone la posición del gobierno británico sobre estas cuestiones y cuya consulta aconsejamos. Ver en: http://www.ukoln.ac.uk/services/lic/newlibrary/html

27. YIP, K. F. Selecting Internet resources. En: The Electronic Library, 15, 2, p. 91-108.

28. NAVELSAKER, T. Selection criteria/scope for Legal Deposit of electronic documents. En: Legal Deposit, with special reference to the archiving of electronic materials. Helsingfors: NORDINFO, 1995. 
29. Mc GOWAN, op. cit., p. 28.

30. WILLE, N. E. Legal Deposit of electronic publications: questions of scope and criteria for selection. Helsingfors: NORDINFO, 1995 (BLR\&DD Report 6197). p. 64-80.

31. FAGERLI, H. M. Access to electronic dynamics documents: a solution to the Legal Deposit problem. Helsingfors: NORDINFO, 1995 (BLR\&DD Report 6197, p. 108-118). Citado por Mc Gowan, op. cit., p. 30). 